\title{
Producing Collisions for PANAMA, Instantaneously
}

\author{
Joan Daemen and Gilles Van Assche \\ STMicroelectronics, Zaventem, Belgium \\ gro.noekeon@noekeon.org
}

\begin{abstract}
We present a practical attack on the PANAMA hash function that generates a collision in $2^{6}$ evaluations of the state updating function. Our attack improves that of Rijmen and coworkers that had a complexity $2^{82}$, too high to produce a collision in practice. This improvement comes mainly from the use of techniques to transfer conditions on the state to message words instead of trying many message pairs and using the ones for which the conditions are satisfied. Our attack works for any arbitrary prefix message, followed by a pair of suffix messages with a given difference. We give an example of a collision and make the collision-generating program available. Our attack does not affect the PANAMA stream cipher, that is still unbroken to the best of our knowledge.
\end{abstract}

Keywords: symmetric cryptography, hash function, collision.

\section{Introduction}

A cryptographic hash function maps a message of arbitrary length to a fixedsize output called a digest. One of the requirements for a cryptographic hash function is collision-resistance: it should be infeasible to to find two different messages that give the same digest.

PANAMA can be used both as a hash function and as a stream cipher. In the scope of this paper, we will consider only on the hash part. Our attack does not have impact on the security of the PANAMA stream cipher. Internally, PANAMA has a state and a buffer, which evolve using a state updating function. For every block of message, the state updating function transforms the state and the buffer. We describe PANAma in Sec. 2

In this article, we describe a method to produce collisions for the PANAMA hash function that refines the method of Rijmen and coworkers 2] and reduces the workload from $2^{82}$ to $2^{6}$ applications of the state updating function. We can therefore generate collisions quasi instantaneously. Furthermore, there are many degrees of freedom in the produced messages. The attack works for any initial value of the state. This means that one can find a collision with a pair of messages $\left(M_{1}\left|M, M_{1}\right| M^{*}\right)$ with an arbitrary prefix $M_{1}$. Here, the message parts $M$ and $M^{*}$ have a fixed difference $M^{\prime}=M+M^{*}$. Furthermore, the attacker 
can append an arbitrary suffix $M_{2}$ to both collision messages, independently of $M_{1}, M$ and $M^{*}$. We discuss the structure of the attack in Sec. 3 .

Like in 2, we use a differential trail (called differential path in 2]) that leads to a zero difference in state and buffer. A differential trail specifies both the message differences and the differences in the state and in the buffer. For a pair of messages to follow the right differences in the state, a subset of the state bits must satisfy specific conditions. In the attack in [2, part of these conditions were transferred to equations on message words while the remaining ones were satisfied by trying many different message pairs and picking out those for which these conditions happened to be satisfied. In our attack, we transfer all conditions to equations on message bits using some simple new techniques explained in Sec. 4. The transfer of equations has negligible workload.

Although very similar, our trail is different from that of [2]. We chose a trail such that the conditions on the state are more easily transferable to equations on the message bits. We describe it in Sec. 5 and all its conditions and their transfer in Sec. 6 .

\section{Description of PANAMA}

The internal memory of PANAMA is composed of 273 32-bit words (hereby denoted words) and is organized in two parts: [1]

- the state, with 17 words denoted $a_{0}$ through $a_{16}$, and

- the buffer, which is an array of $32 \times 8$ words, denoted $b_{i, j}$ with $0 \leq i \leq 31$ and $0 \leq j \leq 7$. (Note that $b_{i}$ indicates a block of 8 words $b_{i, 0} \ldots b_{i, 7}$.)

The + sign applied on bits denotes the exclusive or (xor) operation and on words the bitwise xor. In subscripts of the state $a$, it denotes modulo- 17 addition.

The message to hash is padded and divided into blocks of 8 words (i.e., 256 bits) each. It is processed as follows. First, both the state and the buffer are initialized to 0 . Then, for each message block $p=\left(p_{0}, p_{1}, \ldots, p_{7}\right)$ (i.e., for each round), the following operations are applied:

- the state undergoes a non-linear transformation $\theta \circ \pi \circ \gamma$, with

$$
\begin{aligned}
\gamma: & a_{i} \leftarrow a_{i}+\left(a_{i+1}+\overline{0}\right) a_{i+2}+\overline{0}, \\
\pi & : \quad a_{i} \leftarrow a_{7 i \bmod 17} \ggg i(i+1) / 2, \\
\theta & : \quad a_{i} \leftarrow a_{i}+a_{i+1}+a_{i+4},
\end{aligned}
$$

where the invisible multiplication indicates the bitwise and, $\overline{0}$ denotes the word with 32 bits 1 , and $\ggg$ cyclic right shift of the bits within a word;

- the least significant bit of $a_{0}$ is flipped: $a_{0} \leftarrow a_{0}+1$;

- the message block is xored into the state:

$$
a \leftarrow a+f_{\mathrm{i} \rightarrow \mathrm{s}}(p) \Leftrightarrow a_{i+1} \leftarrow a_{i+1}+p_{i}, 0 \leq i \leq 7
$$


- eight words of the buffer are xored into the state:

$$
a \leftarrow a+f_{\mathrm{b} \rightarrow \mathrm{s}}\left(b_{16}\right) \Leftrightarrow a_{i+9} \leftarrow a_{i+9}+b_{16, i}, 0 \leq i \leq 7 ;
$$

- the buffer undergoes a linear feedback shift register (LFSR) step:

$$
\begin{aligned}
b_{i} & \leftarrow b_{i-1} \bmod 32 \quad(i \neq 25), \\
b_{25} & \leftarrow b_{24}+r\left(b_{31}\right),
\end{aligned}
$$

where the function $r$ is defined as $Y=r(X) \Leftrightarrow Y_{j}=X_{j+2} \bmod 8$;

- the message block is xored into the buffer: $b_{0, i} \leftarrow b_{0, i}+p_{i}, 0 \leq i \leq 7$.

After all the message blocks are processed, 33 extra rounds are performed, called blank rounds. These rounds use the state updating function, with the difference that a part of the state (instead of a message block) is input into the buffer: $b_{0, i} \leftarrow b_{0, i}+a_{i+1}, 0 \leq i \leq 7$.

Finally, the digest is extracted from the state after the blank rounds.

\section{Structure of the Attack}

The first thing to note is that the presence of the blank rounds makes it hard to produce a collision in the digest if there is a difference in either the state or the buffer after all the message blocks are input. Due to the invertibility of the state updating function such a difference will not cancel out. Moreover, the lack of external input and the propagation properties of the state updating function give the attacker almost no control over the final difference. Therefore, our goal is to produce a collision in both the state and the buffer before the blank rounds.

We produce a collision by following a trail. Two instances of PANAMA process two different messages $(p$ and $p+d p)$, which have a given difference $(d p)$. The trail also specifies the differences in the state $(d a)$ and in the buffer $(d b)$ between the two instances of PANAmA, at each round. So, not only the two messages must have the given difference, they must also produce the right difference in the state and in the buffer.

We shall now describe the general structure of the trail used in the scope of this article. We will first talk about the sequence of message differences, then about the differences in the state.

In the sequel, the round numbers are specified between brackets in superscript: ${ }^{(i)}$. The convention is that $p^{(i)}$ is the message block processed during round $i$, and $a^{(i)}$ is the value of the state after round $i$.

\subsection{Collision in the Buffer}

The buffer evolves independently from the state and is linear. As noticed in [2], the following message difference sequence gives a collision in the buffer for any $x$ :

$$
d p^{(1)}=x, d p^{(8)}=r(x), d p^{(33)}=x \text {, all other differences } 0 .
$$


After 32 rounds, we have $d b_{24}=r(x)$ and $d b_{31}=x$. After the 33rd round, we get:

$$
\begin{gathered}
d b_{25} \leftarrow d b_{24}+r\left(d b_{31}\right)=r(x)+r(x)=0, \\
d b_{0} \leftarrow d b_{31}+d p^{(33)}=x+x=0 .
\end{gathered}
$$

Thanks to the linearity of the buffer, any combination of shifted instances of the sequence (1) results in a collision in the buffer. In [2], two such sequences are used, one distant of two rounds from the other. In this paper, we instead use three such sequences at three consecutive rounds. More precisely, the message sequence is as follows (only non-zero differences are indicated):

$$
\begin{array}{ll}
\left(d p^{(1)}, d p^{(2)}, d p^{(3)}\right) & =\left(d^{(1)}, d^{(2)}, d^{(3)}\right), \\
\left(d p^{(8)}, d p^{(9)}, d p^{(10)}\right) & =\left(r\left(d^{(1)}\right), r\left(d^{(2)}\right), r\left(d^{(3)}\right),\right. \\
\left(d p^{(33)}, d p^{(34)}, d p^{(35)}\right) & =\left(d^{(1)}, d^{(2)}, d^{(3)}\right) .
\end{array}
$$

\subsection{Collision in the State}

The state is influenced both by the message blocks and by the buffer words in $b_{16}$. Let us summarize the sequence of differences that are xored into the state, both from the message block and from $b_{16}$ :

I Rounds $r=i+0$ : State gets difference $d p^{(r)} \quad=d^{(i)}$

II Rounds $r=i+7$ : State gets difference $d p^{(r)} \quad=r\left(d^{(i)}\right)$

III Rounds $r=i+17$ : State gets difference $d b_{16}^{(r)}=d p^{(r-17)}=d^{(i)}$

IV Rounds $r=i+24$ : State gets difference $d b_{16}^{(r)}=d p^{(r-17)}=r\left(d^{(i)}\right)$

V Rounds $r=i+32$ : State gets difference $d p^{(r)} \quad=d^{(i)}$

with $1 \leq i \leq 3$.

After the three rounds in each of the five sequences described above, we will make sure that we have a collision in the state. These are called subcollisions. After the last subcollision, we have both a collision in the state and in the buffer, and we are thus guaranteed to obtain the same digest after the blank rounds.

Before we explain how to obtain a subcollision, we need to detail the properties of the difference propagation in $\gamma$, the only non-linear operation of the state updating function.

\subsection{Difference Propagation Through $\gamma$}

Since $\gamma$ is composed only of bitwise operations, we will only talk about $\gamma$ as if it operates on 17 bits in this current subsection. The actual $\gamma$ on words can be seen as 32 such operations in parallel.

Assume that the input of one instance of $\gamma$ is $a$, while the input of the other instance is $a+d a$. For a given input difference $d a$, not all output differences are possible. The output difference $d c=\left(d c_{0}, \ldots, d c_{16}\right)$ is determined by the following equation:

$$
d c_{i}=\gamma_{i}(d a)+d a_{i+1} a_{i+2}+d a_{i+2} a_{i+1}+1,
$$

where $\gamma_{i}(a)=a_{i}+\left(a_{i+1}+1\right) a_{i+2}+1$ denotes a particular output bit of $\gamma$. 
Hence, we can obtain an output difference $d c$ from a given input difference $d a$ only if $a$ satisfies some conditions. These are as follows:

$$
\begin{aligned}
& \text { If } d a_{i+1}=1 \text { and } d a_{i+2}=0 \text {, then } a_{i+2}=d c_{i}+\gamma_{i}(d a)+1 \\
& \text { If } d a_{i+1}=0 \text { and } d a_{i+2}=1 \text {, then } a_{i+1}=d c_{i}+\gamma_{i}(d a)+1 \\
& \text { If } d a_{i+1}=1 \text { and } d a_{i+2}=1 \text {, then } a_{i+1}+a_{i+2}=d c_{i}+\gamma_{i}(d a)+1 \text {. }
\end{aligned}
$$

We call conditions of type (2) and (3) simple conditions and conditions of type (44) two-bit parity conditions. We call a differential $(d a, d c)$ for which the set of conditions has a solution a possible differential.

Note that the input difference $d a$ fully determines the positions of the state bits $a_{i}$ that are subject to conditions. Assume that we have $n$ consecutive 1 s in the pattern $d a$, i.e., we have $d a_{i}=d a_{i+n+1}=0$ and in between $d a_{i+l}=1$ $(1 \leq l \leq n)$. Then there are simple conditions on $a_{i}$ and on $a_{i+n+1}$, and $n-1$ two-bit parity conditions on $a_{i+l}+a_{i+l+1}(1 \leq l<n)$. This can be applied to all such patterns in $d a$.

From this follows that the number of conditions is equal to the Hamming weight of $d a$ plus the number of 001 patterns in $d a$. (For the particular case of $d a=11111111111111111$, there are 16 independent two-bit parity conditions.) We denote by $w(d a)$ the number of conditions due to $d a$.

\subsection{Specifying the Trail}

For our attack to work, we wish to determine equations on the message bits that imply the five subcollisions. In the previous subsection we have shown that given a possible differential $(d a, d c)$ over $\gamma$, we obtain conditions on input bits of $\gamma$.

Consider now subcollision I. Before the first round, there is no difference in the state, hence $d a^{(0)}=0$. At the input of the second round, the message difference appears in the state: $d a^{(1)}=f_{\mathrm{i} \rightarrow \mathrm{s}}\left(d^{(1)}\right)$. This determines the input difference of $\gamma$ in round 2. We now need to specify the output of $\gamma$ in the second round, but we can equivalently specify $d a^{(2)}$, as the other operations are linear. After the third round, the fact that we have a collision in the state imposes that $d a^{(3)}=0$, yielding at the output of the third round a difference equal to $f_{\mathrm{i} \rightarrow \mathrm{s}}\left(d^{(3)}\right)$. Hence a value for $d a^{(2)}$ must be chosen such that differentials $\left(f_{\mathrm{i} \rightarrow \mathrm{s}}\left(d^{(1)}\right), \pi^{-1} \circ \theta^{-1}\left(d a^{(2)}+f_{\mathrm{i} \rightarrow \mathrm{s}}\left(d^{(2)}\right)\right)\right)$ and $\left(d a^{(2)}, \pi^{-1} \circ \theta^{-1}\left(f_{\mathrm{i} \rightarrow \mathrm{s}}\left(d^{(3)}\right)\right)\right)$ over $\gamma$ are possible. For a given message difference sequence $d^{(1)}, d^{(2)}, d^{(3)}$ there may be several, one or none such values of $d a^{(2)}$. Note that the first differential imposes conditions on $a^{(1)}$ and the second one on $a^{(2)}$.

As $\theta$ and $\pi$ are linear, it follows that a possible differential over the stateupdating function imposes conditions on bits of the state $a^{(i)}$. Doing this for differentials over more rounds is more difficult and we avoid it in out attack. Therefore, for each round in which there is non-zero input difference in the state, we need to know the output difference.

For subcollisions II to V, applying the same reasoning leads to following round differentials, which we write as differentials over $\theta \circ \pi \circ \gamma$ for compactness: 


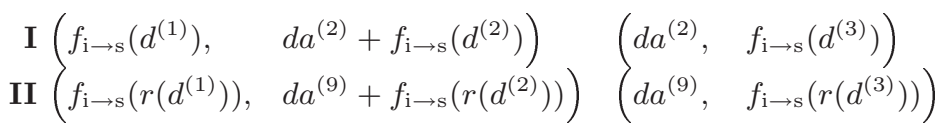

$$
\begin{aligned}
& \text { III }\left(f_{\mathrm{b} \rightarrow \mathrm{s}}\left(d^{(1)}\right), \quad d a^{(19)}+f_{\mathrm{b} \rightarrow \mathrm{s}}\left(d^{(2)}\right)\right) \quad\left(d a^{(19)}, \quad f_{\mathrm{b} \rightarrow \mathrm{s}}\left(d^{(3)}\right)\right) \\
& \mathbf{I V}\left(f_{\mathrm{b} \rightarrow \mathrm{s}}\left(r\left(d^{(1)}\right)\right), \quad d a^{(26)}+f_{\mathrm{b} \rightarrow \mathrm{s}}\left(r\left(d^{(2)}\right)\right)\right)\left(d a^{(26)}, \quad f_{\mathrm{b} \rightarrow \mathrm{s}}\left(r\left(d^{(3)}\right)\right)\right) \\
& \mathbf{V}\left(f_{\mathrm{i} \rightarrow \mathrm{s}}\left(d^{(1)}\right), \quad d a^{(34)}+f_{\mathrm{i} \rightarrow \mathrm{s}}\left(d^{(2)}\right)\right) \quad\left(d a^{(34)}, f_{\mathrm{i} \rightarrow \mathrm{s}}\left(d^{(3)}\right)\right)
\end{aligned}
$$

Hence, the trail is fully determined by the sequence $\left(d^{(1)}, d^{(2)}, d^{(2)}\right)$, and the 5 state differences $d a^{(2)}, d a^{(9)}, d a^{(19)}, d a^{(26)}$ and $d a^{(34)}$. Because the structure of the subcollisions I and $\mathrm{V}$ are equal, we can fix $d a^{(34)}=d a^{(2)}$.

\subsection{Symmetric Patterns}

Like in [2], we use differences with words that are either 0 or $\overline{0}$. This causes the intra-word rotations in $\pi$ to have no influence on the difference pattern, as all other operations in the state updating function work in a bitwise fashion.

Let us translate this in the case of the word-oriented $\gamma$. We can view Equations (22)-(41) as 32 parallel conditions on the bits of the state words. Thanks to the fact that all the difference words $d a_{i}$ are either 0 or $\overline{0}$, the words $a_{j}$ on which these conditions apply are the same for the 32 bits; either all or none of the 32 bits of a word $a_{j}$ are affected by a condition. Hence, the equations can be written word-wise. Note however that this does not restrict the value of the state or of the message words to be either 0 or $\overline{0}$, only the differences.

\section{Techniques for Equation Transfer}

For a given trail, we have seen in Sec. 3.3 how to express conditions on the state $a$ to get the right output differences. In this section, we explain how to transfer these equations to the message words that the attacker can choose.

We will see that the equations are never transferred to more than two rounds before the start of the subcollision, so there is no overlap between the equations derived from different subcollisions and hence we can satisfy them sequentially.

As the discussion below is generic for all five subcollisions, let us use a common convention. For $j \in\{1,8,18,25,33\}$, we denote the various stages of the state transformation with the following symbols:

$$
\begin{aligned}
\stackrel{+p^{(j-2)}}{\longrightarrow} & \mathrm{N} \stackrel{\gamma}{\longrightarrow} \mathrm{O} \stackrel{\theta \circ \pi}{\longrightarrow} \mathrm{P} \stackrel{+p^{(j-1)}}{\longrightarrow} \mathrm{Q} \\
& \mathrm{Q} \stackrel{\gamma}{\longrightarrow} \mathrm{R} \stackrel{\theta \circ \pi}{\longrightarrow} \mathrm{S} \stackrel{+p^{(j)}}{\longrightarrow} \mathrm{T} \\
& \mathrm{T} \stackrel{\gamma}{\longrightarrow} \mathrm{U} \stackrel{\theta \circ \pi}{\longrightarrow} \mathrm{V} \stackrel{+p^{(j+1)}}{\longrightarrow} \mathrm{W} \\
& \mathrm{W} \stackrel{\gamma}{\rightarrow} \mathrm{X} \stackrel{\theta \circ \pi}{\longrightarrow} \mathrm{Y} \stackrel{+p^{(j+2)}}{\longrightarrow} \mathrm{Z} .
\end{aligned}
$$

At a given time, the corresponding italic letter denotes the state value. 
Although we will follow a reasoning going backwards from $\mathrm{Z}$ down to $\mathrm{T}, \mathrm{Q}$ or $\mathrm{N}$, the attack works in practice in the forward direction. As the conditions are being satisfied, the state is updated with the known values.

For each subcollision, we wish to have a collision in the state at time $\mathrm{Z}$, hence to have $d Z=0$. This determines $d Y=f_{\mathrm{i} \rightarrow \mathrm{s}}\left(d p^{(j+2)}\right)$ or $d Y=f_{\mathrm{b} \rightarrow \mathrm{s}}\left(d b_{16}^{(j+1)}\right)$, together with the difference pattern $d X$ via $\pi^{-1} \circ \theta^{-1}$. The trail also specifies the patterns $d W$ and $d T$ (and indirectly $d U$ ). The differential $(d T, d U)$ over $\gamma$ implies conditions on $T$ and $(d W, d X)$ on $W$. The attacker must satisfy them by choosing appropriate values for $p^{(j-2)}, p^{(j-1)}, p^{(j)}$ and $p^{(j+1)}$.

The conditions are either simple, i.e., of type $W_{i}=$ target or two-bit parity conditions $W_{i}+W_{i+1}=$ target where "target" is a known value.

In the sequel, we often speak about the left and right hand sides of an equation. As a convention, the left hand side contains one isolated variable to be solved, while the right hand side contains other variables that are determined from other equations or set to arbitrary values.

\subsection{Immediate Satisfaction in W}

Simple conditions on words $W_{1}$ through $W_{8}$ can be satisfied by setting the value of $p^{(j+1)}$ accordingly. We call this immediate satisfaction:

$$
W_{i}=\text { target } \rightarrow p_{i-1}^{(j+1)}=V_{i}+\text { target }(\text { if } 1 \leq i \leq 8) .
$$

The value of $V_{i}$ is determined by the value of $T$.

A two-bit parity condition $W_{i}+W_{i+1}=$ target can be satisfied whenever (at least) one of the two words can be modified through $p$, that is, when $0 \leq i \leq 8$. For $i=0$ or $i=8$, we have:

$$
\begin{aligned}
& W_{0}+W_{1}=\text { target } \rightarrow p_{0}^{(j+1)}=V_{0}+V_{1}+\text { target }, \text { and } \\
& W_{8}+W_{9}=\text { target } \rightarrow p_{7}^{(j+1)}=V_{8}+V_{9}+\text { target } .
\end{aligned}
$$

If $1 \leq i \leq 7$, however, the value of another message word must be taken into account. This other message word must be treated as known and its value may either be fixed by other conditions or set to an arbitrary value. We have either

$$
\begin{aligned}
& W_{i}+W_{i+1}=\text { target } \rightarrow p_{i-1}^{(j+1)}=V_{i}+V_{i+1}+p_{i}^{(j+1)}+\text { target }, \text { or } \\
& W_{i}+W_{i+1}=\text { target } \rightarrow p_{i}^{(j+1)}=V_{i}+V_{i+1}+p_{i-1}^{(j+1)}+\text { target } .
\end{aligned}
$$

\subsection{Bridge from $\mathrm{W}$ to $\mathrm{T}$}

The conditions on $W$ that cannot be satisfied immediately can be transferred to equations at time $\mathrm{U}$ via $\pi^{-1} \circ \theta^{-1}$.

A condition on some $W_{i}$ can be converted into an equation in three words of $U$. For instance, assume we have to satisfy $W_{10}=0$. We know that

$$
W_{10}=\left(U_{2} \lll 3\right)+\left(U_{9} \lll 45\right)+\left(U_{13} \lll 91\right) .
$$


In this case, $U_{2}$ will be influenced directly by the message words $p^{(j)}$, and this makes it an ideal candidate for immediate satisfaction in T. So, let us isolate this variable and write:

$$
U_{2}=\left(\left(U_{9} \lll 45\right)+\left(U_{13} \lll 91\right)\right) \ggg 3 .
$$

Remember that the terms $U_{9}$ and $U_{13}$ at the right hand side are treated as known values.

In more general terms, a simple condition on $W_{i}$ is converted into an equation on $U_{7 i}^{\pi}+U_{7(i+1)}^{\pi}+U_{7(i+4)}^{\pi}$, with $U_{i}^{\pi}=U_{i} \lll i(i+1) / 2$. One can choose to isolate one of the three variables $U_{7 i}, U_{7(i+1)}$ or $U_{7(i+4)}$. Then, the cyclic rotation on the left hand side can be replaced by its inverse on the right hand side. For instance, the isolation of $U_{7 i}$ gives the following:

$$
W_{i}=\text { target } \rightarrow U_{7 i}=\left(U_{7(i+1)}^{\pi}+U_{7(i+4)}^{\pi}+\text { target }\right) \ggg 7 i(7 i+1) / 2, i \neq 0,
$$

while the constant 1 must be taken care of in the case of the word $i=0$, for instance we can isolate $U_{0}$ as $U_{0}=U_{7}^{\pi}+U_{11}^{\pi}+$ target +1 .

Similarly, a two-bit parity condition on $W_{i}+W_{i+1}$ is converted into an equation on $U_{7 i}^{\pi}+U_{7(i+2)}^{\pi}+U_{7(i+4)}^{\pi}+U_{7(i+5)}^{\pi}$. Again, one can choose to isolate either of the four variables.

An equation of the type $U_{i}=$ target can be written as $T_{i}+\left(\overline{0}+T_{i+1}\right) T_{i+2}=$ target $+\overline{0}$. One can transfer the equation on $T_{i}$ by treating $T_{i+1}$ and $T_{i+2}$ as known values:

$$
U_{i}=\text { target } \rightarrow T_{i}=\left(\overline{0}+T_{i+1}\right) T_{i+2}+\text { target }+\overline{0} .
$$

Combining the substitutions (6) and (7) is called a bridge. Together with the immediate satisfaction, this is the technique we used most often in our collisiongenerating algorithm.

Of course, all the conditions on $T_{1}$ through $T_{8}$ are immediately satisfiable by setting the appropriate value in $p^{(j)}$ as in Equation (5) with $W$ and $V$ replaced by $T$ and $S$, respectively.

\subsection{Side Bridge}

An interesting special case is an equation on $U_{0}=$ target. Since an equation on $T_{0}$ cannot be immediately satisfied via $p_{j}$, we can instead create two equations on $T_{1}$ and $T_{2}$. We can choose from three ways of creating two equations:

$$
\begin{array}{ll}
U_{0}=\text { target } \rightarrow T_{1}=T_{0}+\text { target } & \text { and } T_{2}=\overline{0} \\
U_{0}=\text { target } \rightarrow T_{1}=0 & \text { and } T_{2}=T_{0}+\text { target }+\overline{0} \\
U_{0}=\text { target } \rightarrow T_{1}=T_{0}+\text { target } & \text { and } T_{2}=T_{0}+\text { target }+\overline{0}
\end{array}
$$

In the sequel, this technique is called a side bridge. Note that this technique can also be used on other word positions. 


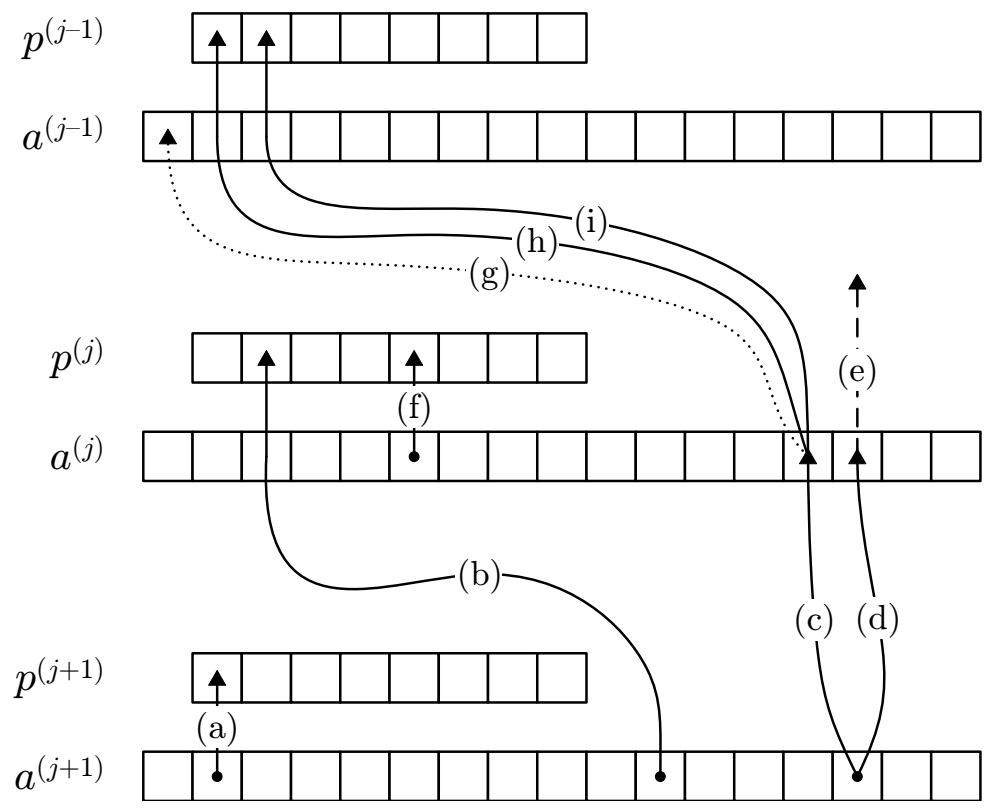

Fig. 1. Schematic illustration of some of the equation transfer techniques. (a) A condition in one of the words $a_{1 \ldots 8}^{(j+1)}$ can be immediately satisfied via $p^{(j+1)}$. (b) Otherwise, this condition has to be bridged to the previous round. Preferably, it is bridged to one of the words $a_{1 \ldots 8}^{(j)}$ so that it can be satisfied via $p^{(j)}$. (c)-(d) Sometimes, a bridge (c) must be accompanied by an additional equation (d) to remove circular dependencies. (e) Of course, this additional equation must be also be bridged or satisfied somewhere (not shown explicitly). (f) Conditions can also appear in round $j$; here is an example of such a condition that can be immediately satisfied via $p^{(j)}$. (g)-(h)-(i) Bridging a condition to the word $0(\mathrm{~g})$ does not allow immediate satisfaction. Instead, it is possible to side-bridge it to two equations, one on word 1 (h) and one on word 2 (i).

\subsection{Dependency Removal}

As said in the beginning of Sec. 4, when solving an equation, all terms on the right hand side must be known. This imposes constraints on the order in which the equations are solved. In some cases, it may be necessary to remove circular dependencies on the way from $U$ to $T$. For instance, assume that we have three equations on $U$ :

$$
\begin{aligned}
& U_{4}=\text { target } \\
& U_{6}=\text { target, } \\
& U_{8}=U_{4}+\text { target (up to rotation). }
\end{aligned}
$$

When transferring from $\mathrm{U}$ to $\mathrm{T}$, (8) requires $T_{5}$ and $T_{6}$ to be known, hence that (9) is already solved for $T_{6}$. In turn, (9) requires $T_{7}$ and $T_{8}$ to be known, hence 
that (10) is solved for $T_{8}$. But (10) requires that $T_{4}$ is known so that (8) must be solved. To remove this circular set of dependencies, we can force $T_{7}=\overline{0}$ so that $U_{6}=T_{6}+\left(T_{7}+\overline{0}\right) T_{8}+\overline{0}=T_{6}+\overline{0}$ does not depend on $T_{8}$ any more. The dependency of (9) on (10) is removed, and we can solve (9), then (8), then (10).

In more general terms, we can set $T_{i+1}=\overline{0}$ (resp. $\left.T_{i+2}=0\right)$ for the equation on $U_{i}=$ target, so that the converted equation on $T_{i}$ has no dependency on $T_{i+2}$ (resp. $T_{i+1}$ ). In the sequel, this technique is called dependency removal. To sum up, one can apply either:

$$
\begin{aligned}
& U_{i}=\text { target } \rightarrow T_{i}=\text { target }+\overline{0} \text { and } T_{i+1}=\overline{0}, \text { or } \\
& U_{i}=\text { target } \rightarrow T_{i}=\text { target }+\overline{0} \text { and } T_{i+2}=0 .
\end{aligned}
$$

The dependency removal and the other techniques are illustrated in Fig. 1.

\subsection{The Conditions Due to Differential $(d T, d U)$}

The differential $(d T, d U)$ fixes some words of $T$. These words are therefore not available for bridges coming from W. Fortunately, there are some degrees of freedom on the way from $\mathrm{W}$ to $\mathrm{T}$ to avoid conflicts. For instance, one should choose adequately the component of $U$ that will be isolated on the left hand side. For the attack to work, there must be a way to satisfy and bridge the equations in a non-conflicting way.

The equations on $T$ in turn can be bridged to equations on $Q$. The conditions on $Q_{1}$ through $Q_{8}$ can then be satisfied via $p^{(j-1)}$.

However, one must pay careful attention to equation dependencies. When for example choosing $p^{(j)}$ to satisfy a condition in $W$, it has an impact on the state at time $\mathrm{T}$ and subsequent rounds. Indeed, changing the value of $T_{i}$ influences the values of $U_{i-2}, U_{i-1}$ and $U_{i}$. Hence care must be taken, when solving equations that are the result of bridges, that equations solved earlier are not affected. In general, dependency problems become more difficult to manage as the number of bridges grows.

\subsection{Solving the Equations by Correction}

When all bridges are determined along with their dependencies, solving the equations is fairly simple and does not involve much more than an evaluation of the state updating function. This is thanks to the fact that the equations are made linear once the right hand side is determined. Let us illustrate this with an example.

Assume that we need to satisfy $W_{9}=0$. Since immediate satisfaction is not possible, we decide to bridge this condition to $T_{6}=$ target (via an equation on $\left.U_{6}\right)$. At this stage, we do not calculate the actual value of target, but we influence $T_{6}$ through $p_{5}^{(j)}$. So, we evaluate the state updating function for round $j$ with $p_{5}^{(j)}=0$ and then the state updating function for round $j+1$. After this, we obtain $W_{9}^{*}$, which may not be zero as we wished. However the linearity 
of the equations implies that we can reuse the value $W_{9}^{*}$ (up to a rotation) to determine $p_{5}^{(j)}$. Since $U_{6}$ is rotated by $6(6+1) / 2=21$ positions, we can simply set $p_{5}^{(j)}=W_{9}^{*} \ggg 21$ and we automatically get $W_{9}=0$.

In short, the linearity allows us to satisfy a condition by correcting the corresponding message word. The right value of the message word is determined (up to a rotation) by the correction to bring to the state word under condition.

The reasoning for satisfying and bridging equations is done backwards in time. In contrast, solving them by correction works forwards in time, and the rounds are evaluated sequentially. The state updating function may be evaluated several times, an extra evaluation being needed for each correction.

The dependencies between equations play an important role in the order in which the corrections are applied. In the same way the right hand side of an equation must be known when solving it, a correction shall not affect equations that have been satisfied earlier.

\section{The Chosen Trail}

To choose a suitable trail, an important parameter to consider is the number of conditions on $T$ and on $W$ in the five subcollisions. Actually, this number should be split in the number of conditions that can be immediately satisfied and those that need to be bridged.

To make a trail easy to exploit using the techniques described in Sec. 4 the application of immediate satisfaction and the bridges coming from the later rounds should as much as possible fit within the 8 message words each round. One can chain bridges over several rounds, but as more rounds are bridged dependencies become increasingly difficult to manage. Generally speaking, the higher the number of conditions to bridge, the more difficult it will be to fit them all on a small number of rounds.

The number of conditions in the first subcollision is $w\left(d a^{(1)}\right)$ in $\mathrm{T}$ and $w\left(d a^{(2)}\right)$ in W. The number of conditions for the other four subcollisions are $\left(w\left(d a^{(i)}\right)\right.$, $\left.w\left(d a^{(i+1)}\right)\right)$ with $i=8,18,25$ and 33 . Note that the fifth subcollision is identical to the first one. We split each of these numbers $w(d a)$ as $w(d a)=w_{\text {is }}(d a)+$ $w_{\mathrm{b}}(d a)$, where $w_{\text {is }}(d a)$ is the number of conditions that can be immediately satisfied and $w_{\mathrm{b}}(d a)$ that must be bridged.

As a heuristic criterion, we minimize the maximum number of conditions to bridge:

$$
W_{\mathrm{b}}=\max _{i}\left\{w_{\mathrm{b}}\left(d a^{(i)}\right)\right\} .
$$

We have searched exhaustively through all $255^{2} 256$ patterns $\left(d^{(1)}, d^{(2)}, d^{(3)}\right)$ and selected the one that has a collision trail with a minimal $W_{\mathrm{b}}$. This resulted in the trail determined by following values:

$-d^{(1)}=00000101, d^{(2)}=11010000, d^{(3)}=01111011$,

$-d a^{(2)}=10111110000000101$,

$-d a^{(9)}=00100011101011000$, 
$-d a^{(19)}=00010111001100000$ and

$-d a^{(26)}=11111100111010010$,

where each digit represents a word either all-zero (0) or all-one (1); the word positions increase from left to right. The number of conditions that can be immediately satisfied or bridged is given in Table 1 below. As a comparison, the trail used in 2] has $W_{b}=7$, while our trail has $W_{b}=5$.

Table 1. Number of conditions in the trail used in our attack

\begin{tabular}{c|cc|cc|cc|cc} 
& \multicolumn{2}{|c|}{$\mathrm{I}$} & \multicolumn{2}{|c|}{$\mathrm{II}$} & \multicolumn{2}{|c|}{$\mathrm{III}$} & \multicolumn{2}{|c}{$\mathrm{IV}$} \\
& $d a^{(1)}$ & $d a^{(2)}$ & $d a^{(8)}$ & $d a^{(9)}$ & $d a^{(18)}$ & $d a^{(19)}$ & $d a^{(25)}$ & $d a^{(26)}$ \\
\hline$w_{\text {is }}$ & 2 & 6 & 3 & 5 & 0 & 5 & 0 & 8 \\
$w_{\mathrm{b}}$ & 1 & 3 & 0 & 4 & 3 & 3 & 3 & 5
\end{tabular}

\section{Equation Transfer in the Chosen Trail}

In this section, we describe in detail how the equations are transferred to the message words using the techniques in Sec. 4 for the trail specified in Sec. 5 .

\subsection{Subcollisions I and V}

For round 1, we have the following differences:

\begin{tabular}{|l|l|}
\hline$d b_{16}^{(0)}=00000000$ & $d a^{(0)}=00000000000000000=d Q$ \\
\hline$d p^{(1)}=00000101$ & Round $\mathbf{1}$ \\
\hline \hline$d a^{(1)}=00000010100000000=d T$ & $d U=00001001100000000$ \\
\hline
\end{tabular}

From the pattern in $d T$, we can see that we have three conditions on $T_{5}, T_{7}$ and $T_{9}$, namely

$$
\begin{aligned}
& T_{5}=d U_{4}+\gamma_{4}(d T)+\overline{0}=0, \\
& T_{7}=d U_{6}+\gamma_{6}(d T)+\overline{0}=d U_{5}+\gamma_{5}(d T)+\overline{0}=0, \\
& T_{9}=d U_{8}+\gamma_{8}(d T)+\overline{0}=\overline{0} .
\end{aligned}
$$

The equations (11) and (12) can be immediately satisfied in round 1 via $p_{4}^{(1)}$ and $p_{6}^{(1)}$, whereas (13) must be bridged to Q:

$$
T_{9}=\overline{0} \rightarrow Q_{2}=\left(\left(\overline{0}+Q_{3}\right) Q_{4}+\overline{0}\right)+\left(R_{6}^{\pi}+R_{12}^{\pi}+\overline{0}\right) \ggg 3,
$$

which can be immediately satisfied in round 0 via $p_{1}^{(0)}$. Note that this implies that $Q_{3}, Q_{4}, \ldots, Q_{8}, Q_{12}, Q_{13}$ and $Q_{14}$ are known when solving for $Q_{2}$. 
Then for round 2, we have the following differences:

\begin{tabular}{|l|l|}
\hline$d b_{16}^{(1)}=00000000$ & \\
\hline$d p^{(2)}=11010000$ & Round 2 \\
\hline \hline$d a^{(2)}=10111110000000101=d W$ & $d X=01110010000001111$ \\
\hline
\end{tabular}

From the pattern in $d W$, this imposes nine conditions on $W_{0}+W_{16}, W_{1}$, $W_{2}+W_{3}, W_{3}+W_{4}, W_{4}+W_{5}, W_{5}+W_{6}, W_{7}, W_{13}$ and $W_{15}$. We will not detail the right hand sides of the corresponding equations, as they can easily be found as explained in Sec. 3.3. The equations on the words $W_{1}$ through $W_{7}$ can be immediately satisfied via $p^{(2)}$. The other equations are solved as follows:

- The condition on $W_{0}+W_{16}$ can be transferred to an equation on $U_{4}^{\pi}+U_{7}^{\pi}+$ $U_{10}^{\pi}+U_{11}^{\pi}$. We isolate $U_{4}$ on the left hand side and transfer it to an equation on $T_{4}$, which can be immediately satisfied via $p_{3}^{(1)}$. (Here, $T_{5}, T_{6}, \ldots, T_{13}$ must be known.)

- The condition on $W_{15}$ is transferred to an equation on $U_{3}^{\pi}+U_{10}^{\pi}+U_{14}^{\pi}$, from which we isolate $U_{3}$, transfer it $T_{3}$ and satisfy it via $p_{2}^{(1)}$. Notice that when isolating $T_{3}$, this means putting $T_{4}$ and $T_{5}$ on the right hand side. The value of $T_{4}$ must thus be determined before that of $T_{3}$, hence the condition on $W_{15}$ may only be solved after the one on $W_{0}+W_{16}$. (Here, $T_{4}, T_{5}, T_{10}, T_{11}, T_{12}$, $T_{14}, T_{15}$ and $T_{16}$ must be known.)

- Similarly, the condition on $W_{14}$ becomes an equation on $U_{0}^{\pi}+U_{6}^{\pi}+U_{13}^{\pi}$, then on $U_{6}$, then on $T_{6}$, then on $p_{5}^{(1)}$. (Here, $T_{0}, T_{1}, T_{2}, T_{7}, T_{8}, T_{13}, T_{14}$ and $T_{15}$ must be known.)

Finally in round 3, the differences in the state cancel. We process subcollision V in the same way, using the message blocks 32 rounds later.

\subsection{Subcollision II}

For round 8, we have the following differences:

\begin{tabular}{|l|l|}
\hline$d b_{16}^{(7)}=00000000$ & $d a^{(7)}=00000000000000000=d Q$ \\
\hline$d p^{(8)}=00010100$ & Round 8 \\
\hline \hline$d a^{(8)}=00001010000000000=d T$ & $d U=00100010000000000$ \\
\hline
\end{tabular}

Hence, we have three conditions on $T_{3}, T_{5}$ and $T_{7}$, which can be immediately satisfied via $p_{2}^{(8)}, p_{4}^{(8)}$ and $p_{6}^{(8)}$. This is summarized in the table below.

\begin{tabular}{|c|c|c|c|}
\hline Condition on & via & then on & satisfied via \\
\hline$T_{3}, T_{5}, T_{7}$ & & & $p_{2}^{(8)}, p_{4}^{(8)}, p_{6}^{(8)}$ \\
\hline
\end{tabular}

Then for round 9, we have the following differences: 


\begin{tabular}{|l|l|}
\hline$d b_{16}^{(8)}=00000000$ & \\
\hline$d p^{(9)}=01000011$ & Round 9 \\
\hline \hline$d a^{(9)}=00100011101011000=d W$ & $d X=10101001000011000$ \\
\hline
\end{tabular}

Here we have nine conditions on $W_{1}, W_{3}, W_{5}, W_{6}+W_{7}, W_{7}+W_{8}, W_{9}$, $W_{11}, W_{12}+W_{13}$ and $W_{14}$. As usual, the conditions on $W_{1}$ through $W_{8}$ can be immediately satisfied via $p^{(9)}$.

\begin{tabular}{|l|l|l|l|}
\hline Condition on & via & then on & satisfied via \\
\hline \hline$W_{1}, W_{3}, W_{5}, W_{6}+W_{7}, W_{7}+W_{8}$ & $p_{0}^{(9)}, p_{2}^{(9)}, p_{4 \ldots 6}^{(9)}$ \\
\hline$W_{12}+W_{13}$ & $U_{0}^{\pi}+U_{10}^{\pi}+U_{13}^{\pi}+U_{16}^{\pi}$ & $T_{1}$ and $T_{2}$, using side bridge & $p_{0}^{(8)}$ and $p_{1}^{(8)}$ \\
\hline$W_{9}$ & $U_{2}^{\pi}+U_{6}^{\pi}+U_{12}^{\pi}$ & $T_{6}$ & $p_{5}^{(8)}$ \\
\hline$W_{14}$ & $U_{3}^{\pi}+U_{7}^{\pi}+U_{13}^{\pi}$ & $T_{13}$, with $T_{8}=\overline{0}$ & $p_{7}^{(8)}$ \\
\hline$T_{13}$ & $R_{0}^{\pi}+R_{6}^{\pi}+R_{14}^{\pi}$ & $Q_{6}$ & $p_{5}^{(7)}$ \\
\hline$W_{11}$ & $U_{3}^{\pi}+U_{9}^{\pi}+U_{16}^{\pi}$ & $T_{9}$, with $T_{11}=0$ & \\
\hline$T_{9}$ & $R_{2}^{\pi}+R_{6}^{\pi}+R_{12}^{\pi}$ & $Q_{2}$, with $Q_{1}=\overline{0}$ & $p_{0}^{(7)}$ and $p_{1}^{(7)}$ \\
\hline$T_{11}$ & $R_{3}^{\pi}+R_{9}^{\pi}+R_{16}^{\pi}$ & $Q_{3}$ & $p_{2}^{(7)}$ \\
\hline
\end{tabular}

For the condition on $W_{14}$, we transfer it to an equation on $U_{3}^{\pi}+U_{7}^{\pi}+U_{13}^{\pi}$. We cannot isolate $U_{3}$ or $U_{7}$ and transfer it to $T_{3}$ or $T_{7}$ since we already have an equation on both. Instead, we isolate $U_{13}$ and transfer the condition to $T_{13}$. We also remove the dependency of $U_{7}$ on $T_{9}$ by setting $T_{8}=\overline{0}$, since $T_{9}$ will be needed below.

The condition on $W_{11}$ can be transferred to an equation on $U_{3}^{\pi}+U_{9}^{\pi}+U_{16}^{\pi}$. Since $T_{3}$ is already busy, we instead isolate $U_{9}$ and transfer the condition to $T_{9}$. However, $U_{9}$ also depends on $T_{10}$, which is influenced by $Q_{2}$; but $Q_{2}$ is needed to satisfy the condition on $T_{9}$. To remove this circular dependency, we force $T_{11}=0$ so that $U_{9}=T_{9}+\overline{0}$ does not depend on $T_{10}$ any more.

The condition on $T_{9}$ is bridged to a condition on $Q_{2}$. However, $Q_{2}$ influences $R_{0}$, whose value is needed to solve for $Q_{6}$; but $Q_{6}$ influences $R_{6}$, whose value is needed to solve for $Q_{2}$. We force $Q_{1}=\overline{0}$ so that $R_{0}=Q_{0}+\overline{0}$ no longer depends on $Q_{2}$.

Finally in round 10, the differences in the state cancel.

\subsection{Subcollision III}

For round 18, we have the following differences:

\begin{tabular}{|l|l|}
\hline$d b_{16}^{(17)}=00000101$ & $d a^{(17)}=00000000000000000=d Q$ \\
\hline$d p^{(18)}=00000000$ & Round 18 \\
\hline \hline$d a^{(18)}=00000000000000101=d T$ & $d U=00000000000010011$ \\
\hline
\end{tabular}

We have three conditions on $T_{0}, T_{13}$ and $T_{16}$, which cannot be immediately satisfied in round 18 . 


\begin{tabular}{|l|l|l|l|}
\hline Condition on & via & then on & satisfied via \\
\hline \hline$T_{0}$ & $R_{0}^{\pi}+R_{7}^{\pi}+R_{11}^{\pi}$ & $Q_{7}$ & $p_{6}^{(17)}$ \\
\hline$T_{13}$ & $R_{0}^{\pi}+R_{6}^{\pi}+R_{13}^{\pi}$ & $Q_{6}$ & $p_{5}^{(17)}$ \\
\hline$T_{14}$ & $R_{3}^{\pi}+R_{10}^{\pi}+R_{14}^{\pi}$ & $Q_{3}$ & $p_{2}^{(17)}$ \\
\hline
\end{tabular}

Then for round 19, we have the following differences:

\begin{tabular}{|l|l|}
\hline$d b_{16}^{(18)}=11010000$ & \\
\hline$d p^{(19)}=00000000$ & Round 19 \\
\hline \hline$d a^{(19)}=00010111001100000=d W$ & $d X=01111011100100000$ \\
\hline
\end{tabular}

This implies conditions on $W_{2}, W_{4}, W_{5}+W_{6}, W_{6}+W_{7}, W_{8}, W_{9}, W_{10}+W_{11}$, $W_{12}$.

\begin{tabular}{|l|l|l|l|}
\hline Condition on & via & then on & satisfied via \\
\hline \hline$W_{2}, W_{4}, W_{5}+W_{6}, W_{6}+W_{7}, W_{8}$ & $p_{1}^{(19)}, p_{3 \ldots .}^{(19)}, p_{7}^{(19)}$ \\
\hline$W_{12}$ & $U_{6}^{\pi}+U_{10}^{\pi}+U_{16}^{\pi}$ & $T_{6}$ & $p_{5}^{(18)}$ \\
\hline$W_{9}$ & $U_{2}^{\pi}+U_{6}^{\pi}+U_{12}^{\pi}$ & $T_{2}$, with $T_{4}=0$ & $p_{1}^{(18)}$ and $p_{3}^{(18)}$ \\
\hline$W_{10}+W_{11}$ & $U_{2}^{\pi}+U_{3}^{\pi}+U_{13}^{\pi}+U_{16}^{\pi}$ & $T_{3}$ & $p_{2}^{(18)}$ \\
\hline
\end{tabular}

To solve the conditions on $W_{9}$ and $W_{10}$, we need to remove some dependencies. On the one hand, the condition on $W_{9}$ is transferred to an equation on $U_{2}^{\pi}+$ $U_{6}^{\pi}+U_{12}^{\pi}$, of which we want to isolate $U_{2}$ and transfer to $T_{2}$; this requires to know $T_{3}$ and $T_{4}$. On the other hand, the condition on $W_{10}+W_{11}$ becomes an equation on $U_{2}^{\pi}+U_{3}^{\pi}+U_{13}^{\pi}+U_{16}^{\pi}$; to isolate $U_{3}$ and transfer the equation to $T_{3}$, we need to know $U_{2}$. This dependency can be removed by forcing $T_{4}=0$ so that $U_{2}$ does not depend on $T_{3}$.

Finally in round 20, the differences in the state cancel.

\subsection{Subcollision IV}

For round 25, we have the following differences:

\begin{tabular}{|l|l|}
\hline$d b_{16}^{(24)}=00010100$ & $d a^{(24)}=00000000000000000=d Q$ \\
\hline$d p^{(25)}=00000000$ & Round 25 \\
\hline \hline$d a^{(25)}=00000000000010100=d T$ & $d U=00000000001110100$ \\
\hline
\end{tabular}

We have three conditions on $T_{11}, T_{13}$ and $T_{15}$, which cannot be immediately satisfied in round 25 .

\begin{tabular}{|l|l|l|l|}
\hline Condition on & via & then on & satisfied via \\
\hline \hline$T_{13}$ & $R_{0}^{\pi}+R_{6}^{\pi}+R_{13}^{\pi}$ & $Q_{6}$, with $Q_{2}=0$ & $p_{5}^{(24)}$ and $p_{1}^{(24)}$ \\
\hline$T_{15}$ & $R_{3}^{\pi}+R_{10}^{\pi}+R_{14}^{\pi}$ & $Q_{3}$ & $p_{2}^{(24)}$ \\
\hline$T_{11}$ & $R_{3}^{\pi}+R_{9}^{\pi}+R_{16}^{\pi}$ & side bridge to $Q_{0}=0$ and to $Q_{1}$ & $p_{0}^{(24)}$ for $Q_{1}$ \\
\hline$Q_{0}$ & $O_{0}^{\pi}+O_{7}^{\pi}+O_{11}^{\pi}$ & $N_{7}$ & $p_{6}^{(23)}$ \\
\hline
\end{tabular}


The condition on $T_{11}$ cannot be satisfied neither in round 25 nor in round 24 . As we transfer it to an equation on $R_{3}^{\pi}+R_{9}^{\pi}+R_{16}^{\pi}$, we cannot isolate $R_{3}$ as it would conflict with the condition on $R_{15}$. We instead isolate $R_{16}$ and side-bridge it to $Q_{0}=0$ and to an equation on $Q_{1}$. The equation on $Q_{1}$ can be satisfied in round 24 via $p_{0}^{(24)}$. Consequently, $Q_{0}=0$ must be bridged to round 23 .

To be able to solve for $T_{13}$, we must prevent $Q_{1}$ from influencing $R_{0}$. Hence, we force $Q_{2}=0$.

Then for round 26, we have the following differences:

\begin{tabular}{|l|l|}
\hline$d b_{16}^{(25)}=01000011$ & \\
\hline$d p^{(26)}=00000000$ & Round 26 \\
\hline \hline$d a^{(26)}=11111100111010010=d W$ & $d X=11000101010010000$ \\
\hline
\end{tabular}

This implies conditions on $W_{0}+W_{1}, W_{1}+W_{2}, W_{2}+W_{3}, W_{3}+W_{4}, W_{4}+W_{5}$, $W_{6}, W_{7}, W_{8}+W_{9}, W_{9}+W_{10}, W_{11}, W_{13}, W_{14}$ and $W_{16}$. Among them, the conditions on $W_{1}$ through $W_{8}$ can be immediately satisfied via $p^{(26)}$.

\begin{tabular}{|l|l|l|l|}
\hline Condition on & via & then on & satisfied via \\
\hline \hline$W_{0}+W_{1}, W_{1}+W_{2}, W_{2}+W_{3}, W_{3}+W_{4}, W_{4}+W_{5}, W_{6}, W_{7}, W_{8}+W_{9}$ & $p^{(26)}$ \\
\hline$W_{9}+W_{10}$ & $U_{6}^{\pi}+U_{9}^{\pi}+U_{12}^{\pi}+U_{13}^{\pi}$ & $T_{6}$, with $T_{8}=0$ & $p_{5}^{(25)}$ and $p_{7}^{(25)}$ \\
\hline$W_{13}$ & $U_{0}^{\pi}+U_{6}^{\pi}+U_{13}^{\pi}$ & side bridge to $T_{1}$ and $T_{2}$ & $p_{0}^{(25)}$ and $p_{1}^{(25)}$ \\
\hline$W_{16}$ & $U_{0}^{\pi}+U_{4}^{\pi}+U_{10}^{\pi}$ & $T_{4}$ & $p_{3}^{(25)}$ \\
\hline$W_{11}$ & $U_{3}^{\pi}+U_{9}^{\pi}+U_{16}^{\pi}$ & $T_{3}$ & $p_{2}^{(25)}$ \\
\hline$W_{14}$ & $U_{3}^{\pi}+U_{7}^{\pi}+U_{13}^{\pi}$ & $T_{7}$ & $p_{6}^{(25)}$ \\
\hline
\end{tabular}

In the condition on $W_{9}+W_{10}$, we set $T_{8}=0$ to remove the dependency of $U_{6}$ on $T_{7}$. This way, the value of $T_{7}$ can be determined after that of $T_{6}$.

Note that the condition on $W_{13}$ becomes an equation on $U_{0}^{\pi}+U_{6}^{\pi}+U_{13}^{\pi}$. Since $U_{6}$ is already taken, we isolate $U_{0}$ and side-bridge it to $T_{1}$ and $T_{2}$.

Finally in round 27 , the differences in the state cancel.

\section{Example of Collision and Workload}

We wrote a program that produces collisions based on the trail described in Sec. 5 and using the equation transfer described in Sec. 6 [4. The workload of the collision-generating function is about 65 applications of the state-updating function: 35 for the 35 message inputs and 30 additional ones for the bridges and some XORs.

An example of pair of collision messages is given in Table 2, which was obtained using our program. Each line represents a message block; for each block, the words in hexadecimal must be read from left to right. The first message is given by the hexadecimal digits in Table 2, while the second message is obtained by xoring with $\overline{0}=$ ffffffff all the underlined words. 
Table 2. Example of pair of messages that produce a collision

$p^{(0)}$ 002911b8 f4046c0d 18be4673 67847de2 4ae13b51 3d6c1b7e 2cd6267d 72ae641d

$p^{(1)} 69522 \mathrm{bd} 8$ 5f903d84 25558553 c194e805 1f7427d8 37edf3e4 bc922535 01eb3a6b

$p^{(2)} \quad \underline{0 e 8257 d 3} \quad \underline{2 e a 67 f d 6} 0682 d f 75$ c21387fe caa1b829 ccc994ba 9d03bd1c 00992518

$p^{(3)} 01244898 \quad \underline{305 \mathrm{e} 252 \mathrm{~b}} \underline{440 \mathrm{~d} 462 \mathrm{c}} \underline{491 \mathrm{c} 5 \mathrm{~b} 2 \mathrm{e}} \underline{4 \mathrm{~d} 061 \mathrm{f} 8 \mathrm{~b}} 4 \mathrm{db} 745 \mathrm{f} 9$ 15473f0e $54 \mathrm{de} 79 \mathrm{dc}$

$p^{(4)}$ 39b355bc 2d1261f0 074d4fca 4dc8390e 6443663d 66bb5f6d 428b7e94 26a61a31

$p^{(5)} 701 \mathrm{f} 5092$ 5d037474 7a5a4baf 767d758d 450940b5 12383ea4 3b253990 1e1f71d5

$p^{(6)}$ 6e5d785e 1ad4176a 63cb2040 6bfc19fc 7f965d80 7ff57876 4e455002 323b054b

$p^{(7)} 24168 c 78$ d6646fb1 9a2ac8f2 030a45b1 301c3921 e58d996a 56ae7f7d 0732105a

$p^{(8)}$ 69bd59fc 6e3b4bdf 1adc0aac 22ee5482 4062e4cf 85f91c0a 45b21fe0 f25f2094

$p^{(9)}$ d7992b2c 1a491c5e 8dc2afaf 3bf6154e a8ab7031 797d40fa $\underline{475 d 1 e f 4}$ e842e121

$p^{(10)} \underline{4 \text { cad0094 }} \underline{314 \mathrm{f} 2 \mathrm{~b} 74} \underline{5 \mathrm{e} 14301 \mathrm{~d}} 4 \mathrm{df21075} \underline{494469 \mathrm{e} 5}$ 2e405ddc $13667210 \underline{1 \mathrm{~cd} 05258}$

$p^{(11)}$ 366b5346 66c441da 42305df2 7eb75e5b 60327a81 2c3b3ba0 15a12e7f $\overline{54220 \mathrm{e} 5 \mathrm{c}}$

$p^{(12)}$ 3ef673cb 0822691d 59913a36 409d0de9 12e16f49 798b6174 121f0502 73da3555

$p^{(13)} 58 \mathrm{~b} 077 \mathrm{~d} 2$ 26ca08ac 3699151a 09021b0b 7bb90ef7 57724ba9 139d0f26 70494f23

$p^{(14)} 692 \mathrm{c} 4 \mathrm{a} 404 \mathrm{a} 00585 \mathrm{~b} 187 \mathrm{e} 5 \mathrm{da} 3$ 16c57533 689955b9 3cd52635 13 e96788 40803068

$p^{(15)} 5 \mathrm{db} 27 \mathrm{fad} 33 \mathrm{ea} 2 \mathrm{e} 1$ 23c91a2a 48cc15d5 575331b2 60bf1732 5c674a5d 3cd6190a

$p^{(16)}$ Ofbf7ae5 2f14185a 6ad630dc 047e26e9 $422 \mathrm{~d} 0 \mathrm{f} 77$ 54dc195d 368e05eb 0d6662b5

$p^{(17)} 7983616975$ ef70c5 2cae43f4 2c49396c 3c613693 e13226d5 5bc5e69d 288f3f57

$p^{(18)} 3 \mathrm{a} 615 \mathrm{feb} 58 \mathrm{~d} 1 \mathrm{~d} 14 \mathrm{c} 00795183$ c49baa76 5e9d7604 79f7f59b $19166 \mathrm{db} 2$ 617207a2

$p^{(19)} 6 \mathrm{~b} 723 \mathrm{ed} 5 \mathrm{f} 5 \mathrm{fe} 7 \mathrm{f} 4 \mathrm{e}$ 401d5fa4 9acbcbfe $038420 \mathrm{bc}$ e3aac878 202e7da1 5b28d301

$p^{(20)} 440246 \mathrm{c} 318 \mathrm{~d} 7068 \mathrm{f}$ 6be842d6 5039652a 542c5a21 19530314 6bcb5da9 0fc946d4

$p^{(21)}$ 0e127504 5f1e7011 $2833660178742718249 \mathrm{e} 328 \mathrm{~d} 2 \mathrm{~b} 0 \mathrm{c} 3 \mathrm{dae} 11 \mathrm{f} 406 \mathrm{bb} 5 \mathrm{dd} 55373$

$p^{(22)}$ 6ad4001c 5a9f6260 4cd460ca 5fa41c20 205913cf 127e075d 003555d6 07cf042f

$p^{(23)} 67322 \mathrm{c} 45 \quad 6 \mathrm{~d} 2259531 \mathrm{af} 46629$ 0ecc37d7 46cf7da8 $01 \mathrm{~d} 35159$ b428c608 3a2d3d0d

$p^{(24)}$ 4fe67839 304d058a 9ffce0a5 09751255 37e6124e 24851e01 591d2784 252a2fd9

$p^{(25)}$ 23c3189f 3362c465 d6437d3f d4bccbbe 507872ed f78a65dd aaa618d1 556224c8

$p^{(26)} 581$ ecd2f 305c16ce 83fde1d9 6b9f1da2 7a1f06d4 efbfe9b6 5fdcde8c 018136fc

$p^{(27)}$ 0c7b1785 50052d8e 0c153981 380717b0 773b727d 06334fbf 728245ee 251f74b1

$p^{(28)} 1 \mathrm{~d} 1842 \mathrm{e} 462705 \mathrm{~d} 85$ 34927fe9 19da7c12 50646f07 4d546b8b 39ce12c6 3bb12fec

$p^{(29)} 4 \mathrm{c} 852466513 \mathrm{e} 15 \mathrm{e} 2 \quad 6 \mathrm{~d} 697 \mathrm{ca} 36 \mathrm{a155ef3} \quad 4 \mathrm{ff} 85 \mathrm{bb} 9$ 5c4603f4 $486 \mathrm{a} 529030046806$

$p^{(30)} 17965 \mathrm{e} 32$ 5e7368b9 470e0ff4 73d95c04 1f163002 182f532d 4d67752c 596871e0

$p^{(31)}$ 4ad4041e 2cf76301 3f4a3974 0a4a00f8 5ed01d43 4e573590 4f68140b 587675a2

$p^{(32)} 66 \mathrm{fa} 4037$ aa864c4d 49bb6092 6f117408 74ad1b53 4eae041c 5d2462b5 05881d37

$p^{(33)} 5579473$ e 7cfe6737 ebed6b2a 912f3f6a dd8bfb4b 329eae68 96076905 6f3c52cc

$p^{(34)}$ 06e8849c 5f456809 102bfd9d 527ab906 a1d33100 72aa5ea1 8ab21c2b 68f50f55

$p^{(35)} 45 \mathrm{c52997} \underline{\text { 39607312 }}$ 345919ca 263d7857$\underline{\text { 3b971002 }}$ 40276cb6 138a726c 29593908

hash result:

$h(p) \mid 45 d 93522$ 0168bdcd e830f65a 6e46f3e9 1bb0bbd6 3d37a576 $718 f 4032$ 0c65079f

\section{Conclusions}

In this paper, we have explained how to refine the attack 2] in order to produce collisions in PANAMA using only about $2^{6}$ evaluations of the state updating 
function. As noted in [2], PANAma gives too many degrees of freedom per round to the attacker.

One could consider to fix PANAMA to be resistant against this type of attack. We have actually done this in [3] and the result is RADIOGATÚN. Its design was based on the insight obtained from the attack in [2] and the possibility of the attack in this paper. This lead us to reduce the number of message words injected each round from 8 to 3 , giving an attacker much less freedom per round and requiring more bridges with accompanying dependency problems for a trail with similar complexity. More importantly, we have added feedback from the state to the buffer, making the buffer evolution during hashing become nonlinear. This makes the split in nicely separated subcollisions no longer possible. For more explanations on the evolution from PANAMA to RADIOGATÚN we refer to Appendix A of [3].

Interestingly, our attack on PANAMA can be seen as an application of trail backtracking 3. In this context, we have defined a metric of a trail called its backtracking depth. As we explain in [3], the backtracking depth gives a good idea of the number of rounds that must be bridged at the worst point in the trail. The backtracking depth of the trail we used in this paper turns out to be only 2. This suggests that the conditions can be satisfied at any round in the trail by the message words injected immediately before it and those before the previous round and hence that the number of bridges is rather limited. In the design of RADIOGATÚN one of the main criteria is exactly the non-existence of collision trails with low backtracking depth.

\section{References}

1. Daemen, J., Clapp, C.S.K.: Fast hashing and stream encryption with PANAMA. In: Vaudenay, S. (ed.) FSE 1998. LNCS, vol. 1372, pp. 60-74. Springer, Heidelberg (1998)

2. Rijmen, V., Van Rompay, B., Preneel, B., Vandewalle, J.: Producing Collisions for PANAMA. In: Matsui, M. (ed.) FSE 2001. LNCS, vol. 2355, pp. 37-51. Springer, Heidelberg (2002)

3. Bertoni, G., Daemen, J., Peeters, M., Van Assche, G.: "RADIOGATÚN a Belt-andMill Hash Function", presented at the NIST Second cryptographic hash workshop (August 2006) available from http://radiogatun.noekeon.org/

4. Program to generate collisions for PANAMA: available from http://radiogatun.noekeon.org/panama 